\title{
Diseño y Validación de una Escala de Competencia Comunicativa y Social para Niños ${ }^{1}$
}

\section{Design and Validation of a Scale of Communicative and Social Skill for Children}

\author{
Ramón D. Castillo \\ Marcelo G. Cancino
}

\author{
Claudia P. Pérez-Salas \\ Joselinne Catalán \\ Universidad de Talca, Chile \\ (Rec: 14 de noviembre 2007 Acep: 04 noviembre 2008)
}

\author{
Carolina Bravo \\ Hedy C. Acosta
}

\section{Resumen}

Se exponen los resultados del diseño y validación de la escala de competencia comunicativa y social (ECCSO) en 1673 niños chilenos entre 9 y 15 años de edad (669 de sexo masculino y 1004 de sexo femenino). El alfa de Cronbach del formato para niños, padres y profesores fue de $0.93,0.95$ y 0.97 respectivamente. El análisis factorial exploratorio con el formato para niños arrojó 8 factores que explican el 30.16\% de la varianza. La ECCSO logró predecir el 50\% de la variabilidad de los puntajes de la Escala de Autoestima de Rosenberg. La validez convergente fue calculada para aquellos niños $(n=52)$ que fueron evaluados simultáneamente por sus padres y profesores; encontrándose moderada convergencia entre niños y padres; divergencia entre niños y profesores; y nula convergencia entre profesores y padres. Para el puntaje total en la ECCSO las niñas obtuvieron mejores puntajes que los niños y no se observaron diferencias por curso y el efecto de interacción entre el sexo y la edad no fue significativo.

Palabras clave: Competencia, lenguaje, pragmática y habilidades sociales.

\begin{abstract}
This article show results about design and validation of the communicative and social competence scale (ECCSO) in 1673 Chilean children, between 9 and 15 years old (669 male and 1004 female sex). Cronbach's alpha for children, parents and teachers formats were 0.93, 0.95 and 0.97 respectively. Exploratory Factor Analysis (EFA) was made for children's format showing 8 factors to explain $30.16 \%$ of variance. The ECCSO could predict $50 \%$ of variability in the scores of Rosenberg's Self-Esteem Scale. The convergent validity was calculated for those children $(n=52)$ who were assessed by parents and teachers; finding a moderate convergence between children and parents scores; divergence between children and teachers; and null convergence between teachers and parents. Girls showed a total score in ECCSO higher than boys; differences between grade, and interaction effect between age and sex were not significant.
\end{abstract}

Key words: Competence, language, pragmatic and social skills.

\footnotetext{
1 Correspondencia a: Ramón D. Castillo Guevara, Facultad de Psicología, Universidad de Talca, Avenida Lircay s/n. Talca, Chile. Fono: (56 71) 201653. E mail: racastillo@utalca.cl

Los resultados de esta investigación fueron presentados en XXV Congreso Internacional de la AELFA y II Encuentro Iberoamericano de Logopedia, Diplomatura de Logopedia de la Universidad de Granada, en Granada, España, Junio 2006. Agradecemos los comentarios y sugerencias a Sergio E. Chaigneau. Este trabajo es parte del proyecto de investigación VAC 600370 financiado por la Universidad de Talca de Chile.
} 


\section{Introducción}

La competencia social es la evaluación global de la capacidad de una persona para interactuar en un contexto interpersonal determinado, y las habilidades sociales son aquellas capacidades específicas necesarias para realizar eficazmente una tarea en dicho contexto. Su evaluación está basada en opiniones de pares, padres o profesores, y con criterios de adecuación culturalmente establecidos (Chou, 1997; Matson, Rotatori \& Helsel, 1983; Méndez, Hidalgo e Inglés, 2002).

En niños y adolescentes, la competencia social apunta al establecimiento de interacciones sociales positivas con pares y adultos, articuladas por destrezas comunicativas (Garaigordobil \& García de Galdeano, 2006; Rinaldi, 2003).

En términos generales, cuando una persona evidencia competencia social, expresa un comportamiento asertivo; escucha a otros; controla sus impulsos; planifica y predice la conducta social; se ajusta a normas; y resuelve conflictos interpersonales (Hidalgo, 2000). Complementariamente, se dice que tiene buena autoestima o autoconcepto; una adecuada capacidad para empatizar, y destrezas para razonar con los estados mentales de los otros (Eisenberg, Wentzel \& Harris, 1998; Garaigordobil \& García de Galdeano, 2006).

Se asocian a la competencia social: la estabilidad emocional, la regulación de emociones, y el buen humor, variables que hacen agradable a la persona, porque la estabilidad y regulación emocional proporcionan consistencia; mientras el buen humor favorece la calidez afectiva en el trato con otros (Buss, 1989; Rinaldi, 2003).

Competencia social y destrezas comunicativas frecuentemente son homologadas, ya que estas últimas, tienen estándares de desempeño que abordan lo lingüístico, paralingüístico y distintos niveles de adecuación pragmática (Caballo, 1993; Puyuelo, Wiig, Renom \& Solanas, 1997; Searle, 1969; Sineiro, Juanatey, Iglesias \& Lodeiro, 2000).

Los componentes lingüísticos son las palabras empleadas, dependientes del rol que se cumple en esa situación y lo que se intenta lograr en ellas. Mientras que los componentes paralingüísticos, son el nivel, intensidad y cambios en el volumen; el tono, o inflexión de la voz y el modo de decir las cosas; la claridad y fluidez en la articulación; el tiempo y velocidad del habla (Caballo, 1993).

Los componentes pragmáticos son la selección y mantención de tópicos, iniciación y cambio de turno, reparo-revisión de quiebres, comentarios de adyacencia y la proporción entre el nivel de cantidad-concisividad y la especificidad-exactitud. También se incluyen los actos de habla, como el uso de palabras interrogativas; las demandas de confirmación o negación; aceptar una crítica justa; rechazar una crítica injusta; mostrar des/aprobación; hacer requerimientos directos e indirectos de acción, entre otros (Caballo, 1993; Martínez, 1995; Puyuelo et al., 1997; Searle, 1969; Sineiro et al., 2000).

Estos están presentes en las interacciones comunicativas, y su uso es esperable en alguien con buenos niveles de competencia social; aún cuando se observan cambios en el desarrollo evolutivo y se reportan diferencias entre hombres y mujeres.

Por ejemplo, se ha establecido que las niñas tienen mayor locuacidad y fluencia del lenguaje que los niños; atribuible a expectativas sociales y diferencias educativas. También se ha constatado que en los juegos de niñas, predominan aspectos interactivos del lenguaje y mayor uso de sustantivos, a diferencia de los juegos de niños, asociados a la acción y la emisión de sonidos (Clemente, 2000).

Mediciones estandarizadas de aspectos pragmáticos del lenguaje, no encontraron diferencias significativas entre niños y niñas (Castillo, Puyuelo, Chaigneau \& Martínez, 2006). Aún cuando estos tenían que ver con actos de habla de carácter más lingüísticos, que no incluían la selección o cambio de tópico, la toma de turno, la variación estilística o los aspectos paralingüísticos, que sí han incluido otros estudios (Sineiro et al., 2000).

Midiendo habilidades sociales también se reportan diferencias entre hombres y mujeres. Por ejemplo, en el desarrollo de una escala de destrezas sociales, los niños presentaban mayores puntuaciones en agresividad y en conducta social inadecuada que las niñas (Matson, Rotatori \& Helsel, 1983). Posteriormente Méndez, Hidalgo e Inglés (2002), estudiando las propiedades psicométricas de esta escala en España, encontraron que las niñas puntuaban con menos agresividad y conducta antisocial; menos presuntuosidad y arrogancia; y más destrezas sociales y asertividad que los niños.

También se ha reportado que las niñas presentan mayor empatía que los niños, una variable moduladora de la conducta prosocial (Garaigordobil \& García de Galdeano, 2006; Mestre, Samper \& Frías, 2002;).

Evolutivamente, los elementos pragmático se desarrollan antes que los fonológicos, sintácticos y semánticos; supuestamente porque es necesario dominar las reglas de uso del lenguaje en contexto social, ya sea para obtener cosas y mantener un sentido de eficacia en la interacción comunicativa (Martínez, 1995). Las mediciones de aspectos pragmáticos, reportan que de los 5 a 14 años su uso mejora (Castillo et al., 2006; Puyuelo et al., 1997;).

Sobre habilidades sociales, no se reporta un incremento de estas durante el desarrollo. Por ejemplo, Matson et al (1983), observaron entre los 4 y 18 años diferencias en la conducta social inapropiada, en niños de 10 años con respecto a los demás grupos. En este estudio, cuando el informe era del profesor, se detectaron diferencias en el puntaje total de destreza social, entre los 6 y 8 años, con respecto a los demás grupos. 
Posteriores estudios con esta escala, no registran diferencias entre los 12 y 17 años, salvo interacción entre la edad y el sexo, en aislamiento-ansiedad social, donde las niñas de 14 años tienen significativamente más puntuación que los niños de esa edad (Méndez et al., 2002).

En resumen, los estudios en competencia social y variables moduladoras como la empatía, dejan en evidencia un mejor desempeño de las mujeres, y no detectan el incremento sostenido que se espera con la edad o escolaridad. Lo contrario se observa en aspectos comunicativos pragmáticos, donde se detecta un incremento sostenido entre distintos tramos de edad, y excepcionalmente reportan diferencias entre hombres y mujeres.

Un análisis de los reactivos de las escalas que miden competencia social deja en claro que lo hacen con descriptores generales (por ejemplo; amistoso, agradable, entre otros) y omiten las conductas específicas de una interacción comunicativa (Rodríguez, Lira, Arancibia \& Bralic, 1996; Silva y Martorel, 1987). Por otro lado, muchas de ellas se centran en conductas desajustadas como ansiedad social o agresividad y no se refieren a la competencia comunicativa (Achenbach \& Edelbrock, 1983; Konold, Walthall, \& Pianta, 2004; Lemos, Fidalgo, Calvo y Menéndez, 1992; Matson et al., 1983).

Lo anterior puede conducir a error en procesos de medición y diagnóstico, ya que un niño o persona con ausencia de dichas conductas desajustadas, no necesariamente es competente socialmente.

Con estos antecedentes, se conceptualizó la competencia social en cuatro dimensiones. La primera, referida a conductas de tolerancia y receptividad a la crítica; entendidas como la capacidad para aceptar comentarios de otras personas; y aprovechar esta retroalimentación en la interacción comunicativa. La segunda, sobre habilidades conversacionales, enfatiza componentes paralingüísticos tales como el volumen y tono de la voz, la claridad y precisión, la consistencia verbal-no verbal, la iniciativa en la toma de turno y los comentarios de adyacencia entre otros. La tercera, sobre conductas de respeto y valoración hacia el interlocutor; y de respeto a la autoridad y normas. Finalmente, la cuarta, de conductas asertivas; para defender derechos, sin agredir o actuar pasivamente.

Con base a este referente, se diseñó una escala para medir competencia comunicativa y social, como autoinforme e informe externo; desechando afirmaciones con descriptores generales o que aludieran a conductas desajustadas.

\section{Método}

\section{Diseño de la ECCSO}

Confección de reactivos: Se redactaron 90 reactivos considerando las definiciones de las cuatro dimensiones y fueron distribuidos en 4 subescalas. La primera de ellas denominada Tolerancia y Receptividad a la Crítica (TRC), la segunda denominada Habilidades Comunicativas (HC), la tercera denominada Conductas Respetuosas y Prosociales (CRP) y la cuarta, denominada Asertividad (AS).

Evaluación por jueces: La ECCSO fue validada por 11 psicólogos (4 doctores, 6 magíster y 1 licenciado). Cada juez independientemente evaluó si el reactivo se adecuaba a la definición conceptual de la subescala a la que pertenecía, y tenía que indicar si el ítem era adecuado, medianamente adecuado o inadecuado. Para cada ítem se calculó el coeficiente de validez de contenido de Cohen, Swerdlik \& Smith (1992); donde un valor igual o mayor a 0,59 con un $95 \%$ de seguridad, hace que un ítem sea considerado válido, cuando es evaluado por 11 jueces. De este proceso, sólo quedaron 60 ítems para la aplicación piloto.

Aplicación piloto: La versión fue aplicada a 57(74\%) niños y $20(26 \%)$ niñas de una escuela de la ciudad de Talca. Segmentados por escolaridad, $43(55,8 \%)$ eran de quinto y 34 (44,2\%) de octavo básico. Se calculó el coeficiente Alfa de Cronbach y fue de 0,94 para la ECCSO, y por subescalas los valores fueron de 0,71 para TRC; 0,86 para HC; 0,83 para CRP y 0,58 para AS. Se eliminaron 8 ítems poco consistentes, quedando la escala con 52 ítems.

\section{Validación de la ECCSO}

Participantes: Participaron 1673 alumnos de escuelas de la ciudad de Talca de Chile. De ellos, 669(40\%) eran niños y $1004(60 \%)$ eran niñas. La media de edad fue de 11,6 años, con una desviación estándar de 1,23 años; con un rango de edad que fluctuó entre los 9 y 15 años. Por curso; $380(22,7 \%)$ eran de quinto básico; $475(28,4 \%)$ de sexto básico; 406 (24,3\%) de séptimo básico y $396(23,7 \%)$ de octavo básico.

Instrumentos: Además de la ECCSO, se aplicó la escala de autoestima de Rosenberg (Rosenberg, 1965). Esta fue usada como criterio de validación de la competencia social, tal como se ha sido usada en otras investigaciones (Chou, 1997).

Procedimientos: Las escalas se administraron el primer semestre del 2005 en forma colectiva, por evaluadores entrenados. Estos leían las instrucciones en voz alta y verificaban que los alumnos contestaran las preguntas. El tiempo promedio de evaluación fue de 50 minutos por curso. Durante el segundo semestre, se entregaron las versiones para padres y profesores, quienes debían evaluar simultáneamente a 270 alumnos. De estas sólo se recuperaron 52 evaluaciones.

\section{Resultados}

Fiabilidad: Se calculó el coeficiente Alfa de Cronbach y los valores de la ECCSO para alumnos, profesores y 
apoderados fueron de $0.93,0.97$ y 0.95 respectivamente. En ese mismo orden, para la subescala TRC, los valores fueron $0.73,0.90$ y 0.86 ; para $\mathrm{HC}$ fueron de $0.86,0.95$ y 0.93; para CRP fueron de $0.79,0.93$ y 0.78 y, finalmente para AS, los valores fueron $0.59,0.81$ y 0.68 . Para la escala de autoestima de Rosenberg, contestada sólo por alumnos, el valor fue de 0.81 .

Validez de constructo: Se hizo un análisis factorial exploratorio, con extracción por factorización de ejes principales y rotación varimax; emergiendo 8 factores que explican el 30,16\% de la variabilidad, con adecuado ajuste $\left(\mathrm{KMO}=0.96 ; \chi^{2}\right.$ de Barlett $\left.=17,039.35 ; \mathrm{gl}=1081 ; \mathrm{p}<0.001\right)$ (Tabla 1).

El primer factor agrupa reactivos de la subescala $\mathrm{HC}$, en especial los comentarios de adyacencia y aclaración del discurso.
El segundo factor contiene reactivos de la subescala TRC; de habilidad para reconocer y enmendar errores; y usar esta retroalimentación en la interacción comunicativa.

Los factores tercero y cuarto poseen reactivos de la subescala CRP; agrupando los reactivos de respeto a la autoridad y las normas sociales; y los reactivos de respeto y tolerancia a los demás como personas respectivamente.

El quinto factor, tiene reactivos de la subescala $\mathrm{HC}$, circunscritos al interés por comprender o empatizar con el otro y adecuarse a su estilo comunicativo.

El sexto factor agrupa reactivos de la subescala AS, enfatizando la habilidad para expresar y recibir críticas.

Los factores séptimo y octavo poseen reactivos de la subescala HC; que dan cuenta de la iniciativa para establecer una conversación; y en el adecuado uso del tono de la voz y la coherencia verbal-no verbal, respectivamente.

Tabla 1: Matriz de factores rotados de la ECCSO

\begin{tabular}{|c|c|c|c|c|c|c|c|c|}
\hline Ítems & F1 & $\mathrm{F} 2$ & F3 & F4 & F5 & F6 & F7 & F8 \\
\hline i14/ Suelo introducir temas relacionados al asunto que se está tratando (HC) & 0.57 & & & & & & & \\
\hline $\begin{array}{l}\text { i17/ Hago comentarios que se relacionan con lo que se está diciendo en el } \\
\text { momento (HC) }\end{array}$ & 0.48 & & & & & & & \\
\hline $\begin{array}{l}\text { i15/ Hago comentarios y/o preguntas apropiadas en conversaciones grupales } \\
\text { (HC) }\end{array}$ & 0.44 & & & & & & & \\
\hline i26/ Cuando tengo una idea, la expreso en forma clara $(\mathrm{HC})$ & 0.41 & & & & & & & \\
\hline i19/ Expreso con precisión mis necesidades a los demás (HC) & 0.37 & & & & & & & \\
\hline i13/ Planteo preguntas relacionadas con la conversación (HC) & 0.37 & & & & & & & \\
\hline $\begin{array}{l}\text { i54/ Frente a un conflicto con otra persona, defiendo mi posición con argu- } \\
\text { mentos claros (AS) }\end{array}$ & 0.36 & & & & & & & \\
\hline i21/ Mis gestos coinciden con lo que quiero comunicar (HC) & 0.35 & & & & & & & 0.32 \\
\hline i7/ Frente a una equivocación, busco activamente soluciones (TRC) & 0.35 & 0.33 & & & & & & \\
\hline $\begin{array}{l}\text { i37/ Cuando no logro darme a entender, intento explicar lo mismo con otras } \\
\text { palabras o mediante ejemplos (HC) }\end{array}$ & 0.31 & & & & & & & \\
\hline i30/ Soy capaz de expresar verbalmente lo que pienso y siento (HC) & * & & & & & & & \\
\hline $\begin{array}{l}\text { i58/ Cuando pasan a llevar mis derechos, expreso mi disgusto de forma apro- } \\
\text { piada (AS) }\end{array}$ & * & & & & & & & \\
\hline i24/ Mantengo la atención de otros cuando estoy hablando (HC) & $*$ & & & & & & & \\
\hline i6/ En un trabajo grupal asumo mis errores y trato de corregirlos (TRC) & & 0.47 & & & & & & \\
\hline i45/ Acepto las decisiones que toman los adultos (TRC) & & 0.46 & & & & & & \\
\hline $\begin{array}{l}\text { i5/ Tengo la capacidad de cambiar mi conducta en base a las sugerencias } \\
\text { realizadas por adultos (TRC) }\end{array}$ & & 0.43 & & & & & & \\
\hline $\mathrm{i} 4$ / Me muestro interesado por reparar mis errores (TRC) & & 0.41 & & & & & & \\
\hline i2/ Acepto y reconozco mis equivocaciones (TRC) & & 0.38 & & & & & & \\
\hline $\begin{array}{l}\text { i60/ Si cometo un error en el que involucro (afecto) negativamente a otros, } \\
\text { pido disculpas (AS) }\end{array}$ & & 0.33 & & & & & & \\
\hline $\begin{array}{l}\text { i12/ Cuando se disgustan conmigo, me esfuerzo por comprender que conducta } \\
\text { mía pudo haber contribuido al disgusto (TRC) }\end{array}$ & & $*$ & & & & & & \\
\hline $\begin{array}{l}\text { i8/ Acostumbro a rescatar lo positivo de las críticas que otros me hacen } \\
\text { (TRC) }\end{array}$ & & $*$ & & & & & & \\
\hline i31/ Miro a los ojos cuando me hablan (HC) & & $*$ & & & & & & \\
\hline
\end{tabular}


i40/ Suelo respetar las reglas al interior de la sala de clases (CRP)

$\begin{array}{cc} & 0.58 \\ 0.30 & 0.44 \\ & 0.42 \\ & 0.39 \\ & 0.35 \\ & 0.33 \\ & *\end{array}$

i51/ Hablo respetuosamente con mis mayores (CRP)

i47/ Obedezco las normas impuestas por la autoridad (CRP)

0.42

i41/ Demuestro capacidad para adaptarme a las normas (CRP)

0.39

i23/ Me mantengo atento cuando otro habla (HC)

0.35

i10/ Cuando recibo una mala calificación demuestro interés por saber en qué me equivoqué (TRC)

i56/ Cuando alguien me insulta, le comunico calmadamente que no me gusta que me traten de esa forma (AS)

i46/ En situaciones de debate soy capaz de respetar las diferentes opiniones (CRP)

i9/ Acepto adecuadamente las críticas de mis compañeros (TRC)

i53/ En trabajos grupales, acepto las decisiones del grupo aunque sean diferentes a mi postura original (CRP)

i43/ Me muestro respetuoso frente a las normas preestablecidas por el grupo (CRP)

i38/ Escucho a los otros aún cuando no comparta su opinión (HC)

i48/ Manifiesto respeto por las diferencias (étnicas, religiosas, políticas y sociales) de otras personas (CRP)

i28/ Al conversar con otros, intento comprender lo que me están diciendo (HC)

i36/ Soy capaz de cambiar mis palabras dependiendo de con quién estoy hablando (compañeros- niños menores, adultos, familiares) (HC)

i20/ Cuando me dicen algo, demuestro interés por comprender lo comunicado (HC)

i59/ Expreso en forma respetuosa mi descontento con algunas tareas que se me encomiendan (AS)

i52/ Al dirigirme a mis compañeros adopto una actitud respetuosa y cordial (AS)

i34/ Soy capaz de iniciar conversaciones con compañeros del sexo opuesto (HC)

i33/ Soy capaz de iniciar conversaciones con compañeros del mismo sexo (HC)

i35/ Soy capaz de iniciar conversaciones con adultos (HC)

i18/ El tono de mi voz coincide con lo que deseo comunicar (HC)

0.47

$0.46 \quad 0.38$

0.45

0.37

*

0.47

0.38

0.33

0.38

0.34

0.34

0.60

0.57

$*$

0.41

0.34

(HC)

i22/ Modifico el tono de mi voz para hacer más entendible el mensaje (HC)

i25/ Al hablar de mis sentimientos, mi cuerpo y mi cara reflejan lo que estoy diciendo (HC)

Valores propios

(\% de varianza)

$\begin{array}{llllllll}3.02 & 2.37 & 2.18 & 1.67 & 1.39 & 1.25 & 1.22 & 1.09\end{array}$

\begin{tabular}{llllllll}
$6.4 \%$ & $5.0 \%$ & $4.6 \%$ & $3.6 \%$ & $3.0 \%$ & $2.7 \%$ & $2.6 \%$ & $2.3 \%$ \\
\hline
\end{tabular}

* Cargas factoriales inferiores a 0,30

Validez predictiva: Con un análisis de regresión múltiple, donde las subescalas fueron predictores del puntaje en la escala de autoestima de Rosenberg; el modelo predijo el $50 \%$ de la variabilidad $\left(\mathrm{R}=0.71 ; \mathrm{F}_{[4,1668]}=417.37 ; \mathrm{p}<0,001\right)$. Las subescalas se relacionaron positivamente con la autoestima. La subescala HC se relacionó más $(\beta=0.46 ; \mathrm{t}=16.86$; $\mathrm{p}<0.001)$; seguida de CRP $(\beta=0.18 ; \mathrm{t}=6.25 ; \mathrm{p}<0.01)$; en tercer lugar $\mathrm{AS}(\beta=0.08 ; \mathrm{t}=3.29 ; \mathrm{p}<0.01)$ y finalmente TRC $(\beta=0.06 ; \mathrm{t}=2.07 ; \mathrm{p}<0.01)$.
Validez convergente: Se calculó la correlación de Pearson entre las tres versiones de la ECCSO (Tabla 2). Observándose que las evaluaciones de apoderados y alumnos convergen parcialmente, que hay nula convergencia entre profesores y apoderados; y hay divergencia entre alumnos y profesores.

Comparación por sexo y escolaridad: Por medio de un análisis de varianza factorial se evaluaron posibles diferencias por sexo y escolaridad (Tabla 3). En el puntaje total, las 
Tabla 2: Correlaciones de Pearson ( $\mathrm{n}=52)$ entre la versiones alumnos(as) (AL), profesores (PR) y apoderados (AP) en la ECCSO y sus subescalas.

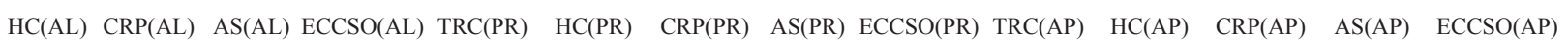

\begin{tabular}{|c|c|c|c|c|c|c|c|c|c|c|c|c|c|c|}
\hline TRC(AL) & $0.68 * *$ & $0.71 * *$ & $0.64 * *$ & $0.84 * *$ & -0.19 & -0.12 & 0.03 & 0.21 & 0.12 & 0.19 & 0.13 & $0.31 *$ & 0.14 & -0.02 \\
\hline $\mathrm{HC}(\mathrm{AL})$ & & $0.72 * *$ & $0.66^{* *}$ & $0.94 * *$ & $-0.25^{*}$ & -0.13 & -0.01 & 0.19 & 0.05 & $0.23 *$ & 0.13 & 0.21 & 0.19 & 0.01 \\
\hline CRP(AL) & & & $0.65 * *$ & $0.87 * *$ & -0.19 & -0.14 & 0.08 & 0.18 & 0.08 & $0.27 *$ & 0.22 & $0.32 *$ & 0.20 & 0.13 \\
\hline $\mathrm{AS}(\mathrm{AL})$ & & & & $0.79 * *$ & $-0.25^{*}$ & $-0.23 *$ & 0.10 & 0.19 & 0.00 & 0.20 & 0.11 & $0.24 *$ & 0.11 & -0.02 \\
\hline $\operatorname{ECCSO}(\mathrm{AL})$ & & & & & $-0.24 *$ & -0.15 & 0.03 & 0.20 & 0.07 & $0.24 *$ & 0.16 & $0.27 *$ & 0.18 & 0.03 \\
\hline TRC(PR) & & & & & & 0.19 & 0.18 & 0.01 & 0.16 & -0.07 & -0.04 & 0.07 & -0.11 & -0.18 \\
\hline HC(PR) & & & & & & & $0.32 * *$ & -0.15 & 0.12 & 0.06 & 0.05 & 0.22 & 0.04 & 0.05 \\
\hline CRP(PR) & & & & & & & & -0.03 & 0.04 & -0.13 & -0.07 & 0.20 & -0.16 & -0.05 \\
\hline $\mathrm{AS}(\mathrm{PR})$ & & & & & & & & & $0.34 * *$ & 0.16 & 0.15 & 0.12 & 0.07 & 0.00 \\
\hline $\mathrm{ECCSO}(\mathrm{PR})$ & & & & & & & & & & 0.09 & 0.14 & 0.19 & 0.00 & -0.03 \\
\hline TRC(AP) & & & & & & & & & & & $0.37 * *$ & 0.17 & $0.35^{* *}$ & $0.49 * *$ \\
\hline HC(AP) & & & & & & & & & & & & $0.28 *$ & 0.20 & $0.34 * *$ \\
\hline CRP(AP) & & & & & & & & & & & & & 0.22 & -0.01 \\
\hline $\mathrm{AS}(\mathrm{AP})$ & & & & & & & & & & & & & & $0.23 *$ \\
\hline
\end{tabular}

niñas tienen mejores puntajes que los niños $\left(\mathrm{F}_{[1,1649]}=48.16\right.$; $\mathrm{p}<0.001 ; 1-\beta=0.99)$. Por curso no se aprecian diferencias $\mathrm{y}$ tampoco se observan efectos de interacción entre el sexo y la escolaridad.

Para las subescalas TRC $\left(\mathrm{F}_{[1,1649]}=29.03 ; \mathrm{p}<0.001\right.$; $1-\beta=0.99) ; \mathrm{HC}\left(\mathrm{F}_{[1,1649]}=29.99 ; \mathrm{p}<0.001 ; 1-\beta=0.99\right), \mathrm{CRP}$ $\left(\mathrm{F}_{[1,1649]}=42.38 ; \mathrm{p}<0.001 ; 1-\beta=0.99\right)$ y AS $\left(\mathrm{F}_{[1,1649]}=79.78\right.$; $\mathrm{p}<0.001 ; 1-\beta=0.99)$ se encontraron diferencias por sexo, donde nuevamente las niñas tienen mejores puntajes que los niños. Para las subescalas TRC $\left(\mathrm{F}_{[3,1649]}=3.98 ; \mathrm{p}<0.01\right.$; $1-\beta=0.84) ; \mathrm{HC}\left(\mathrm{F}_{[3,1649]}=3.10 ; \mathrm{p}<0.05 ; 1-\beta=0.73\right)$ y $\mathrm{CRP}$ $\left(\mathrm{F}_{[3,1649]}=3.33 ; \mathrm{p}<0.05 ; 1-\beta=0.76\right)$ se observaron diferencias por curso; donde los de quinto y sexto año en conjunto tienen puntuaciones significativamente mayores a los de séptimo y octavo. No se observaron efectos de interacción entre el sexo y la escolaridad. En la escala de autoestima de Rosenberg no se encontró ningún efecto significativo. Dadas las diferencias entre niños y niñas, se hicieron normas diferenciadas (Tabla 4).

\section{Discusión}

Se conceptualizó la competencia social y comunicativa en cuatro dimensiones específicas, enfatizando destrezas y omitiendo la referencia a conductas desajustadas o a descriptores globales. Para cada dimensión se construyeron reactivos que, una vez validados por jueces, dieron origen a una escala, con un formato para alumnos, profesores y apoderados.

La escala es fiable en sus tres formatos, con valores iguales o mayores a 0.93 . Las subescalas presentan adecuados niveles de consistencia, salvo la subescala $\mathrm{AS}$ en el formato de alumnos, que nos hace pensar que la definición de asertividad debe ser readecuado y descrito desde la perspectiva de los mismos alumnos ya que se presume que algunas de las conductas descritas como asertivas en el contexto escolar, en especial la defensa enfática de derechos, son vistas como agresivas y eso repercute en una menor consistencia.

La solución factorial obtenida mantiene concordancia parcial con la teoría, ya que hay cuatro factores que desagregan las habilidades comunicativas en comentarios de adyacencia y aclaración del contenido del discurso; el interés por comprender al otro y adecuarse a su estilo comunicativo; la iniciativa para establecer una conversación y el adecuado uso del tono de la voz y la coherencia verbal-no verbal. Los demás factores mantienen los reactivos básicos de la tolerancia y receptividad a la crítica, las conductas de respeto y prosociales y la asertividad.

La validez predictiva de la ECCSO permite explicar el $50 \%$ de la variabilidad del autoestima; y de estas, habilidades comunicativas se constituye en el mejor predictor. Esta afirmación se hace comparando los resultados de validación de otro estudio, donde la relación con la escala de autoestima de Rosenberg fue de 0.34 y la varianza explicada fue de un 12\% (Chou, 1997).

Los resultados de la validez convergente son preliminares, ya que sólo los profesores y padres de 52 alumnos respondieron. Con la evidencia actual, los profesores tienen una valoración opuesta de las competencias que se atribuyen los alumnos y entre los padres y alumnos existe un nivel bajo de convergencia. De mantenerse esta tendencia en los protocolos, se optará por trabajar sólo con la escala del alumno y del apoderado, tal como lo han hecho otros inventarios o escalas, que no contemplan una versión de los profesores (Kadesjö et al., 2004). 
Tabla 3: Medias y errores típicos para niños y niñas entre $5^{\circ}$ y $8^{\circ}$ de enseñanza básica $(\mathrm{N}=1657)^{*}$

\begin{tabular}{|c|c|c|c|c|c|c|c|c|}
\hline \multirow[b]{2}{*}{ Escalas } & \multicolumn{2}{|c|}{$\begin{array}{l}5^{\circ} \text { Básico } \\
(\mathrm{n}=380)\end{array}$} & \multicolumn{2}{|c|}{$\begin{array}{c}6^{\circ} \text { Básico } \\
(\mathrm{n}=475)\end{array}$} & \multicolumn{2}{|c|}{$\begin{array}{l}7^{\circ} \text { Básico } \\
(\mathrm{n}=406)\end{array}$} & \multicolumn{2}{|c|}{$\begin{array}{c}8^{\circ} \text { Básico } \\
(n=396)\end{array}$} \\
\hline & $\begin{array}{l}\text { Niños } \\
(\mathrm{n}=150)\end{array}$ & $\begin{array}{c}\text { Niñas } \\
(\mathrm{n}=230)\end{array}$ & $\begin{array}{l}\text { Niños } \\
(\mathrm{n}=188)\end{array}$ & $\begin{array}{c}\text { Niñas } \\
(\mathrm{n}=287)\end{array}$ & $\begin{array}{l}\text { Niños } \\
(\mathrm{n}=172)\end{array}$ & $\begin{array}{c}\text { Niñas } \\
(\mathrm{n}=234)\end{array}$ & $\begin{array}{l}\text { Niños } \\
(\mathrm{n}=172)\end{array}$ & $\begin{array}{c}\text { Niñas } \\
(\mathrm{n}=245)\end{array}$ \\
\hline $\begin{array}{l}\text { Escala de competen- } \\
\text { cia comunicativa y } \\
\text { social (ECCSO) }\end{array}$ & $175.02(2.22)$ & $187.30(1.79)$ & $178.79(1.98)$ & 190.62(1.61) & $176.19(2.09)$ & $184.20(1.77)$ & $178.63(2.22)$ & $184.10(1.74)$ \\
\hline $\begin{array}{l}\text { Tolerancia y recep- } \\
\text { tividad a la crítica } \\
\text { (TRC) }\end{array}$ & $33.43(0.48)$ & $35.76(0.38)$ & $33.79(0.42)$ & $35.68(0.34)$ & $33.05(0.44)$ & $34.59(0.38)$ & $33.26(0.47)$ & $33.83(0.37)$ \\
\hline $\begin{array}{l}\text { Habilidades comuni- } \\
\text { cativas (HC) }\end{array}$ & $85.39(1.17)$ & $90.91(0.94)$ & $88.19(1.04)$ & $93.62(0.84)$ & $87.35(1.09)$ & $90.51(0.93)$ & $89.48(1.16)$ & $91.13(0.91)$ \\
\hline $\begin{array}{l}\text { Conducta de respeto } \\
\text { y prosocial (CRP) }\end{array}$ & $38.81(0.55)$ & $41.16(0.45)$ & $39.05(0.49)$ & $41.56(0.40)$ & $38.06(0.52)$ & $40.24(0.44)$ & $38.16(0.55)$ & $40.00(0.43)$ \\
\hline Asertividad (AS) & $17.39(0.32)$ & $19.48(0.26)$ & $17.57(0.29)$ & $19.79(0.23)$ & $17.63(0.30)$ & $19.02(0.26)$ & $17.73(0.32)$ & $19.15(0.25)$ \\
\hline $\begin{array}{l}\text { Escala de autoestima } \\
\text { de Rosenberg }\end{array}$ & $39.18(0.59)$ & $39.72(0.47)$ & $39.81(0.53)$ & $41.62(0.43)$ & $40.40(0.55)$ & $39.65(0.47)$ & $40.77(0.59)$ & $40.06(0.46)$ \\
\hline
\end{tabular}

Tabla 4: Normas diferenciadas para niños y niñas.

\begin{tabular}{|c|c|c|c|c|c|c|c|c|c|c|}
\hline \multirow[b]{2}{*}{ Percentiles } & \multicolumn{5}{|c|}{ Niños } & \multicolumn{5}{|c|}{ Niñas } \\
\hline & $\begin{array}{c}\text { Tolerancia y } \\
\text { receptividad } \\
\text { a la crítica } \\
\text { (TRC) }\end{array}$ & $\begin{array}{c}\text { Habilidades } \\
\text { comunicativas } \\
\text { (HC) }\end{array}$ & $\begin{array}{c}\text { Conducta } \\
\text { de respeto } \\
\text { y prosocial } \\
\text { (CRP) }\end{array}$ & $\begin{array}{l}\text { Asertividad } \\
\text { (AS) }\end{array}$ & $\begin{array}{c}\text { Escala de } \\
\text { competencia } \\
\text { comunicativa } \\
\text { y social } \\
\text { (ECCSO) } \\
\end{array}$ & $\begin{array}{c}\text { Tolerancia y } \\
\text { receptividad } \\
\text { a la crítica } \\
\text { (TRC) }\end{array}$ & $\begin{array}{c}\text { Habilidades } \\
\text { comunicativas } \\
(\mathrm{HC})\end{array}$ & $\begin{array}{c}\text { Conducta } \\
\text { de respeto } \\
\text { y prosocial } \\
(\mathrm{CRP})\end{array}$ & $\begin{array}{l}\text { Asertividad } \\
\text { (AS) }\end{array}$ & $\begin{array}{c}\text { Escala de } \\
\text { competencia } \\
\text { comunicativa } \\
\text { y social } \\
\text { (ECCSO) }\end{array}$ \\
\hline 10 & $7-26$ & $15-69$ & $10-30$ & $4-12$ & 44-142 & $15-27$ & $19-74$ & $10-33$ & $5-14$ & $55-155$ \\
\hline 20 & $27-29$ & $70-76$ & $31-33$ & $13-14$ & $143-154$ & $28-30$ & $75-81$ & $32-36$ & $15-16$ & $156-168$ \\
\hline 30 & $30-31$ & $77-81$ & $34-36$ & $15-16$ & $155-165$ & $31-32$ & $82-86$ & $37-38$ & $17-18$ & $169-176$ \\
\hline 40 & 32 & $82-86$ & $37-38$ & 17 & $166-174$ & $33-34$ & $87-90$ & $39-40$ & 19 & $177-183$ \\
\hline 50 & $33-34$ & $87-90$ & 39 & 18 & $175-181$ & $35-36$ & $91-93$ & $41-42$ & 20 & 184-189 \\
\hline 60 & 35 & $91-93$ & $40-41$ & 19 & $182-188$ & 37 & $94-97$ & 43 & 21 & $190-196$ \\
\hline 70 & $36-37$ & $94-96$ & $42-43$ & 20 & 189-194 & 38 & $98-100$ & $44-45$ & 22 & $197-202$ \\
\hline 80 & $38-39$ & $97-100$ & $44-45$ & 21 & $195-200$ & $39-40$ & $101-103$ & 46 & 23 & 203-209 \\
\hline 90 & $40-45$ & $101-155$ & $46-50$ & $22-25$ & $201-262$ & $41-45$ & 104-155 & $47-50$ & $24-25$ & $210-262$ \\
\hline Media (DE) & $33,41(6,04)$ & $87.64(14.78)$ & $38.53(7.01)$ & $17.60(4.13)$ & $177.18(28.39)$ & $35.01(5.62)$ & $91.69(13.84)$ & $40.79(6.48)$ & $19.39(3.79)$ & $186.88(25.99)$ \\
\hline
\end{tabular}

En todas las dimensiones de la ECCSO las niñas presentan mejores puntajes que los niños, lo que es consistente con el comportamiento de las escalas que miden competencia y ajuste social, sin embargo no se aprecian diferencias en los niveles de autoestima (Garaigordobil \& García de Galdeano, 2006; Matson et al., 1983; Mestre et al., 2002).

Las leves diferencias por escolaridad que se observan, posiblemente se deben a que los niños de séptimo y octavo se encuentren entre los 11 y 14 años, edades donde otros estudios han mostrado un mayor nivel de desajuste social, atribuible a los cambios en la adolescencia (Kadesjö et al., 2004; Lemos et al., 1992; Matson et al., 1983; Méndez et al., 2002).

En resumen, se cuenta con una escala para evaluar la competencia comunicativa y social de escolares a partir de sus propias percepciones; con niveles de fiabilidad y validez adecuados, sensible a las diferencias por sexo y con versiones preliminares para padres y profesores, las cuales se encuentran en proceso de validación.

Esta escala es complementaria a los instrumentos vigentes, que tienden a enfatizar el déficit o desajuste social; y añade criterios conductuales explícitos, en vez de descriptores globales para evaluar el desempeño.

Las siguientes fases de nuestra investigación, apuntan a evaluar los niveles de sensibilidad y especificidad de la escala con muestras clínicas y de control, además de analizar la convergencia con dispositivos que evalúan funciones cognitivas y lenguaje en contextos naturales y de laboratorio.

\section{Referencias}

Achenbach, T. M. \& Edelbrock, C. S. (1983). Manual for the Child Behavior Checklist and Revised Child Behavior Profile. Burlington, VT: University of Vermont. 
Buss, A. (1989). Temperaments as personality traits. En: G. A. Kohnstamm, J.E. Bates \& M. Rothbart (Eds), Temperament in childhood (pp. 49-58). Chichester: John Wiley.

Caballo, V. (1993). Manual de evaluación y entrenamiento de habilidades sociales. Madrid: Editorial Siglo XXI.

Castillo, R.; Puyuelo, M., Chaigneau, S. \& Martínez, L. (2006). Validación de los módulos semántico y pragmático de la Batería de Lenguaje Objetiva y Criterial (BLOC) en niños Chilenos. Psicothema, 18(2), 326-332.

Chou, K.L. (1997). The Matson of social skills with youngsters: realibility and validity of a Chinese translation. Personality and individual differences, 22(1), 123-125.

Clemente, R. (2000). Desarrollo del lenguaje, manual para profesionales de la intervención en ambientes educativos. Barcelona: Ediciones Octaedro.

Cohen, R. J., Swerdlik, M. E., \&Smith, D. K. (1992). Psychological testing and assessment: An introduction to tests and measurements. Mountain View, CA: Mayfield Publishing Company.

Eisenberg, N., Wentzel, M. \& Harris, J. (1998). The role of emotionality and regulation in empathy-related responding. School Psychology Review, 4, 506-521.

Garaigordobil, M. \& García de Galdeano, P. (2006). Empatía en niños de 10 a 12 años. Psicothema, 18(2), 180-186.

Hidalgo, C. G. (2000). Comunicación interpersonal, programa de entrenamiento en habilidades sociales. Santiago de Chile: Ediciones Universidad Católica de Chile.

Kadesjö, B., Janols, L., Korkman, M., Mickelsson, K., Strand, G., Trillingsgaard, A. \& Gillberg, C. (2004). The FTF (Five to Fifteen): the development of a parent questionnaire for the assessment of ADHD and comorbid conditions. European Child Adolescent Psychiatry, 13, 3-13.

Konold, T., Walthall, J. \& Pianta, R. (2004). The behavior of child behavior ratings: Measurement structure of the Child Behavior Checklist across time, informants, and child gender. Behavioral Disorders, 29 , $372-383$.
Lemos, S., Fidalgo, A. M., Calvo, P. \& Menéndez, P. (1992). Salud mental de los adolescentes Asturianos. Psicothema, 4(1), 21-46.

Matson, J., Rotatori, A. \& Helsel, W. (1983). Development of a ranting scale to measure social skills in children: The Matson evaluation of social skills with youngsters (MESSY). Behaviour Research Therapy, 21(4), 335-340.

Martínez, L. (1995). Pragmática aplicada a la Fonoaudiología. Curso de perfeccionamiento presentado en las XV Jornadas Nacionales de Fonoaudiología. Santiago, Chile.

Méndez, F. X., Hidalgo, M. D. \& Inglés, C. J. (2002). The Matson of social skills with youngsters. European Journal of Psychological Assessment, 18(1), 30-42.

Mestre, M.V., Samper, P. \& Frías, M.D. (2002). Procesos cognitivos y emocionales predictores de la conducta prosocial y agresiva: La empatía como factor modulador. Psicothema, 14(12), 227-232.

Puyuelo, M., Wiig, E., Renom, J. \& Solanas, A. (1997). Batería de Lenguaje Objetiva y Criterial: Manual de Evaluación, Barcelona: Masson, S.A.

Rinaldi, C. (2003). Language competente and social behavior of students with emocional or behavioral disorders. Behavioral Disorders, 29(1), 34-42.

Rodríguez, S., Lira, M., Arancibia, V. \& Bralic, S. (1996). IPCS. Inventario de problemas conductuales y socioemocionales para niños entre 3 y 5 años. Santiago de Chile: Ediciones Universidad Católica de Chile.

Rosenberg, M. (1965). Society and the adolescent self-image. Princeton, NJ: Princeton University Press.

Searle, J.R. (1969). Speech acts: an essay in the philosophy of language. London: Cambridge University Press.

Silva, F. \& Martorell, M.C. (1987). BAS-3. Batería de Socialización. Madrid: TEA.

Sineiro, C., Juanatey, P., Iglesias, M. \& Lodeiro, O. (2000). Problemas socioemocionales y habilidades lingüísticas en preescolares. Psicothema, 12(3), 412-417. 